\title{
RESHIMMING OF TEVATRON DIPOLES; A PROCESS-QUALITY AND LESSONS-LEARNED PERSPECTIVE*
}

\author{
J.N. Blowers\# , J.A. Carson, R.W. Hanft, D.J. Harding, R.W. Robotham \\ FNAL, Batavia, IL 60501, U.S.A.
}

\begin{abstract}
Over the last two years corrections have been made for the skew quadrupole moment in 530 of the 774 installed dipoles in the Tevatron. This process of modifying the magnets in situ has inherent risk of degrading the performance of the superconducting accelerator. In order to manage the risk, as well as to ensure the corrections were done consistently, formal quality tools were used to plan and verify the work. The quality tools used to define the process and for quality control are discussed, along with highlights of lessons learned.
\end{abstract}

\section{BACKGROUND}

In order to provide the context for this work, we briefly provide some background information. Further details can be found in another paper from this conference [1].

\section{Coupling}

In the Tevatron the horizontal and vertical betatron oscillations have been strongly coupled for over a decade, requiring the skew quadrupole correction circuits to run at about $60 \%$ of their capacity ( 15 times stronger than when Tevatron operations began in 1984).

In early 2003, studies were conducted to try and account for the high excitation of the skew quadrupole correctors [2]. The conclusion was that the Tevatron dipoles, on average, had in increase of about one unit of skew quadrupole harmonic as compared to their original magnetic measurements (where a unit is defined as $1 \mathrm{E}^{-4} 0^{-4}$ of the principle field measured at the reference radius).

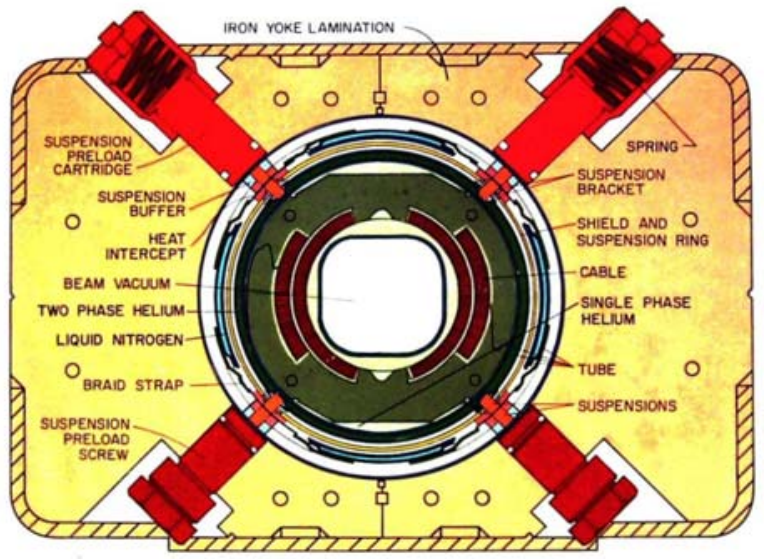

Figure 1: Tevatron Dipole Cross-section

\footnotetext{
* Work supported by the United States Department of Energy under Contract No. DE-AC02-76CH03000.

"blowers@fnal.gov
}

\section{Lift Measurements, Creep \& the Smoking Gun}

The so-called lift measurement is the distance between the upper surface of the suspension preload cartridge and the top surface of the push rod inside the cartridge. Since the cartridges are hollow, the lift can be measured using a depth probe.

In 2003, the lifts of $\sim 100$ installed Tevatron dipoles were measured. The original lift data were extracted from the production records, and we found that, on average, the lift had increased by $\sim 6$ mils, which meant the coils dropped an average of $\sim 4$ mils relative to the yoke. This change would result in an increase in the skew quadrupole harmonic of about one unit - we had found our smoking gun! Due to the force applied to the G10/G11 suspensions within the cryostat, over time the material crept, which resulted in the coil assembly dropping relative to the outer yoke. This change in position then caused the skew quadrupole harmonic to increase.

\section{REMEDIATION}

There is a shim pack on each of the suspension preload screws' faces whose thickness can be simply adjusted by removal of the screw. During production in the early 1980's, each magnet was custom shimmed to minimize the skew quadrupole harmonic. It was envisioned that this process, or something very similar, would need to be done again in order to minimize the unwanted harmonic. However, the original shimming was done while the magnets were in the relatively simple environment of being mounted on a test stand in the Magnet Test Facility. For this round of "reshimming" the magnets would be installed in the Tevatron enclosure, which is a more hostile environment for work such as this.

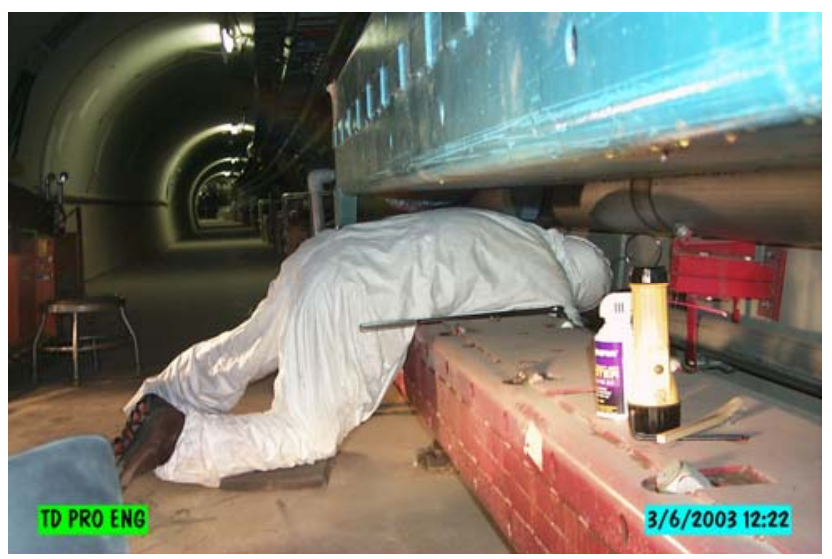

Figure 2: A Technician working on an installed dipole

Due to limitations in the legacy data, we could not reliably confirm the exact change in lift for any single 
magnet, but instead relied upon the population average. Due to this, as well as the fact that what was needed was an average change integrated over the entire ring, it was decided that we would reshim all the magnets the same amount.

In order to help ensure that this work was done successfully and safely, we used formal quality tools to plan and verify the work.

\section{PROCESS QUALITY PLANNING CONSIDERATIONS}

\section{Failure Modes}

The project had two overall goals: 1) make sure the corrections were done correctly, and 2) do not do any harm, to either personnel or magnet. Regarding the first goal, a certain amount of variation could be tolerated (i.e. some stations, or even whole magnets, could be skipped if needed). The second goal was an absolute, and no failure could be tolerated. As such, we needed to understand the various ways in which the process could fail. The tool of choice for this part of the planning is a Process Failure Modes and Effects Analysis (PFMEA). The overall process for creating a PFMEA consists of defining and documenting: the work process, the potential failure modes, the effect(s) if the failure were to occur, the cause(s) of each effect, and the process controls needed to mitigate the causes. The process of developing the necessary controls is often iterative.

Below are highlights from this planning process. The complete PFMEA developed by the reshimming team can be found in an unpublished internal note [3].

\section{Data Collection}

The quality control of the reshimming process depends on accurate lift measurements. Historically this was done by manually recording the lift measurement, and then entering the data by hand into a database. Our experience with hand-entered data has been $\sim 5 \%$ defect rate. With the large amount of data being collected in a very short period of time, we decided to use an automated data collection system called Pocket $M L \AA$. This system used Mitutoyo software and connecting cables, and off-theshelf hardware.

\section{Data Variation}

In order to ensure that our quality control data were accurate, we needed to understand and quantify the natural variation present in the lift measurement process. This was accomplished by conducting gage repeatability and reproducibility $(\mathrm{R} \& \mathrm{R})$ studies. The first study was done to set a baseline. The operators used their own techniques to calibrate the gage and measure the lifts. The result was a two mil R\&R (+/- $3 \sigma)$. Given that we would be looking for a four mil change in lift, we needed to improve on this result. We adopted a standard methodology, trained all the operators, and conducted the second study. The result was a 0.7 mil R\&R, which was much more pleasing.

\section{Quality Assurance}

Central to assuring quality is the use of training, parts kits, travelers, and a correction action system. The benefits of training are self-evident. The benefits of parts kits are two-fold: first we ensure that we have enough parts to complete the project, and second, the counts of parts help to ensure that the reshimming work was done accurately (e.g. parts left over means stations were not reshimmed). We note that if custom shimming were to be done, this would add complexity to the kitting process. The fact that all magnets were to be reshimmed the same amount led to a simplified process of making the kits and assigning them to magnets. Travelers define the specifics of what to do, and in what order to do it. This is critical to ensure that all steps are completed for each magnet. The corrective action system is used to record and track problems which arise during the work. We anticipated encountering unforeseen issues, and this system was very useful for capturing the knowledge gained from correcting them.

\section{Data Analysis (Quality Control)}

In order to verify that each station was appropriately reshimmed, each magnet had two sets of lift measurements taken: one before the shims were added, and then another set after. At the end of each day, these data were uploaded into a database for analysis. The expectation was that we would see approximately a four mil decrease in the lift due to reshimming. Stations which had a non-standard response would be investigated the following day.

\section{Ergonomics}

The successful completion of any physical project depends on healthy personnel. Given the environment in which this work would take place, it was clear that good ergonomics would be critical to ensure that our technicians remained healthy for the duration of the project (774 magnets modified over a three-year period during the annual operational shutdowns lasting 8-12 weeks each). The principle applied was to minimize the physical rigors associated with every step of the reshimming process. Primarily this was accomplished through the tooling supplied. Where one could not avoid physical strain, training was provided to help the technicians understand how best to do the work. The work pace was also defined such that each technician could take frequent breaks.

\section{TECHNICAL RESULTS}

Between the 2003 and 2004 shutdowns, 530 of the 774 installed dipoles have been successfully reshimmed. The effects of the reshimming have been reported in other papers [1][2], but in brief the skew quadrupole correction circuits have been reduced, to first approximation, by the same percentage as the percent of magnets reshimmed 
(i.e. 68\%). In addition, the vertical dispersion has been reduced by 50\% [4].

Figure 3 shows the difference in lift, averaged over all stations for each magnet. There are only 528 data points because two magnets were replaced prior to taking the post reshim lift measurement at liquid helium temperature (i.e. "cold”).

Cold pre reshim - cold post reshim (tunnel) Average (all stations)

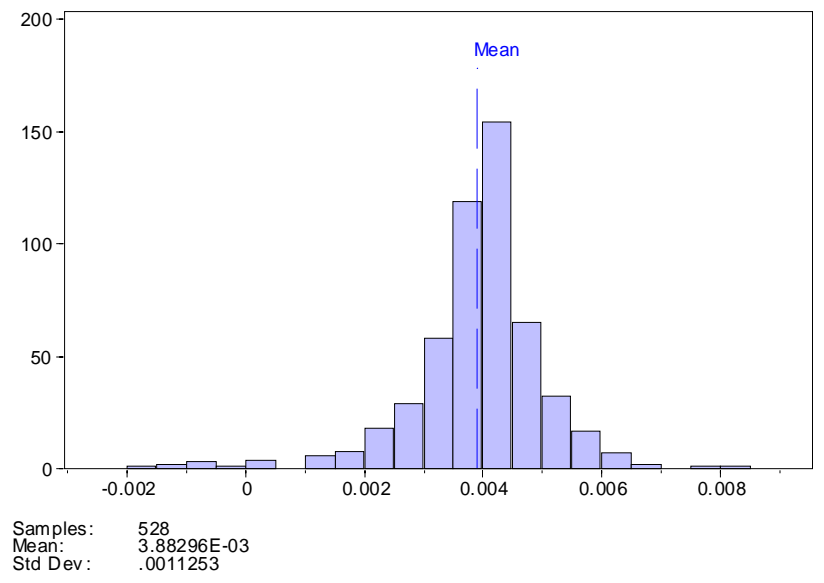

Figure 3: Histogram of Average Lift Change

\section{LESSONS LEARNED}

First, it was very clear that our work planning paid off. To date the project has been very successful. The anticipated failure modes were successfully controlled. This serves as a lesson that the use of formal quality tools can be very effective in planning work. However, not every failure was thought of, and so we encountered a few new problems along the way.

\section{Lift Response}

Some stations had very odd responses to the addition of the shim (at least as was indicated by the pre to post reshim lift change). This was identified in 2003, but it wasn't until 2004 that we were able to connect this issue to a time period of fabrication. We subsequently discovered that we could virtually eliminate the symptom by loosening and retightening all the smart bolts, and then take the pre reshim measurement.

\section{Data Analysis Resources}

Another lesson learned was that we underestimated the resources needed to conduct the data analysis in the first run. In the first run (2003) we had two people (roughly 1.5 full-time equivalents) working on this, and this was not enough to adequately address all the needs in a timely manner. For the second run (2004), this was understood, and so the work planning was done to allow for more time to be spent on the data analysis.

\section{Data Variation}

The lift data taken during the first run showed that the R\&R increased back up to $\sim 2$ mils. This was believed to be partly due to the rigors of the tunnel environment, as well as technician variation. For the second run, the technicians were reminded of the risks associated with the measurement process. This appeared to result in more consistent measurements.

Some of the width in figure 3 can be explained by measurement variation or technical issues related to individual magnets. Despite improvements made between the two runs, the variation remained rather constant, which is not fully understood.

\section{Temperature Effects}

During the first run we were reminded that, although the actual temperature of the magnet does not matter, the temperature must remain constant during the reshimming process. For a small number of magnets the temperatures changed between the pre and post reshim measurement, which resulted in the quality control check for those magnets to be invalid. For the second run, we maintained improved communication between the reshimming teams and the cryogenics crews, and the result was we did not encounter that problem again.

\section{CONCLUSIONS}

In summary, the success to date of this project is the direct result of the excellent personnel doing the work, as well as the detailed planning and use of formal quality tools. We were also very deliberate about remembering what we learned in the first run, and applying it to the second run. Admittedly, there is nothing new or revolutionary about this. The tools and methods described here have been in active use in industry for many decades. There has, however, been some reluctance to use formal quality assurance in basic research. But, when it comes to planning and implementing the business side of basic research, it is hoped that the reader will take away an appreciation that formal quality assurance has its place.

\section{REFERENCES}

[1] D.J. Harding, et al., "Restoring the Skew Quadrupole Moment in Tevatron Dipoles," these proceedings.

[2] M.J. Syphers and D.J. Harding, "Deterioration of the Skew Quadrupole Moment in Tevatron Dipoles Over Time," these proceedings.

[3] J.N. Blowers, et al., "Reshimming Tevatron Dipoles During the Fall 2003 Shutdown," TD-04-004, http://tdserver1.fnal.gov/tdlibry/TD-Notes/.

[4] Norman L. Gelfand, "The Effect of Reshimming of the Tevatron Dipoles (Fall, 2004) to Correct the $a_{1}$ Moment on the Calculated Skew Quadrupole Correctors,” Beams-doc-1489, December 2004, http://beamdocs.fnal.gov/. 